\title{
Overview of the pathological results and treatment characteristics in the first 1000 patients randomized in the SERC trial: axillary dissection versus no axillary dissection in patients with involved sentinel node
}

Gilles Houvenaeghel ${ }^{1,2^{*}}$, Monique Cohen ${ }^{1,2}$, Pédro Raro ${ }^{5}$, Jérémy De Troyer ${ }^{6}$, Christine Tunon de Lara ${ }^{7}$, Pierre Gimbergues ${ }^{8}$, Tristan Gauthier ${ }^{9}$, Christelle Faure-Virelizier ${ }^{10}$, Véronique Vaini-Cowen ${ }^{11}$, Stéphane Lantheaume ${ }^{12}$, Claudia Regis ${ }^{13}$, Emile Darai ${ }^{14}$, Vivien Ceccato ${ }^{15}$, Gauthier D'Halluin ${ }^{16}$, Francesco Del Piano ${ }^{17}$, Richard Villet ${ }^{18}$, Eva Jouve ${ }^{19}$, Bassoodéo Beedassy ${ }^{20}$, Pierrick Theret ${ }^{21}$, Philippe Gabelle ${ }^{22}$, Cécile Zinzindohoue ${ }^{23}$, Pierre Opinel ${ }^{24}$, Catherine Marsollier-Ferrer ${ }^{25}$, Caroline Dhainaut-Speyer ${ }^{26}$, Pierre-Emmanuel Colombo ${ }^{27}$, Eric Lambaudie ${ }^{1,2}$, Agnès Tallet ${ }^{1,3}$, Jean-Marie Boher ${ }^{1,4}$ and Others investigators (SERC trial group)

\footnotetext{
Abstract

Background: Three randomized trials have concluded at non inferiority of omission of complementary axillary lymph node dissection (CALND) for patients with involved sentinel node (SN). However, we can outline strong limitations of these trials to validate this attitude with a high scientific level. We designed the SERC randomized trial (ClinicalTrials.gov, number NCT01717131) to compare outcomes in patients with SN involvement treated with ALND or no further axillary treatment. The aim of this study was to analyze results of the first 1000 patients included.

Methods: SERC trial is a multicenter non-inferiority phase 3 trial. Multivariate logistic regression analysis was used to identify independent factors associated with adjuvant chemotherapy administration and non-sentinel node (NSN) involvement.

Results: Of the 963 patients included in the analysis set, 478 were randomized to receive CALND and 485 SLNB alone. All patient demographics and tumor characteristics were balanced between the two arms. SN ITC was present in 6.3\% patients (57/903), micro metastases in 33.0\% (298), macro metastases in 60.7\% (548) and 289 (34.2\%) were non eligible to Z0011 trial criteria.

Whole breast or chest wall irradiation was delivered in 95.9\% (896/934) of patients, adjuvant chemotherapy in 69.5\%

(Continued on next page)
}

\footnotetext{
*Correspondence: g.houvenaeghel@orange.fr;

houvenaeghelg@ipc.unicancer.fr

'Institut Paoli Calmettes \& CRCM \& Aix Marseille Univ, 232 Bd de Sainte

Marguerite, 13009 Marseille, France

${ }^{2}$ Department of surgery, Institut Paoli Calmettes \& CRCM \& Aix Marseille Univ,

232 Bd Ste Marguerite, Marseille, France

Full list of author information is available at the end of the article
}

(c) The Author(s). 2018 Open Access This article is distributed under the terms of the Creative Commons Attribution 4.0 International License (http://creativecommons.org/licenses/by/4.0/) which permits unrestricted use, distribution, and reproduction in any medium, provided you give appropriate credit to the original author(s) and the source, provide a link to the Creative Commons license, and indicate if changes were made. The Creative Commons Public Domain Dedication waiver (http://creativecommons.org/publicdomain/zero/1.0/) applies to the data made available in this article, unless otherwise stated. 


\begin{abstract}
(Continued from previous page)
(644/926), endocrine therapy in 89.6\% (673/751) and the proportions were similar in the two arms. The overall rate of positive NSN was 19\% (84/442) for patients with cALND. Crude rates of positive NSN according to SN status were $4.5 \%$ for ITC (1/22), 9.5\% for micro metastases (13/137), 23.9\% for macro metastases (61/255) and were respectively 29.36\% (64/218), 9.33\% (7/75) and 7.94\% (10/126) when chemotherapy was administered after CALND, before cALND and for patients without chemotherapy.

Conclusion: The main objective of SERC trial is to demonstrate non inferiority of CALND omission. A strong interaction between timing of CALND and chemotherapy with positive NSN rate was observed.
\end{abstract}

Trial registration: This study is registered with ClinicalTrials.gov, number NCT01717131 October 19, 2012.

Keywords: Breast cancer, Sentinel lymph node biopsy, Axillary lymph node dissection, Randomized trial

\section{Key message}

SERC trial compared outcomes in patients with SN involvement treated with ALND or no further axillary treatment. We analyze results of the first 1000 patients included. Crude rates of positive NSN according to SN status were 4.5, 9.5, 23.9\% for ITC, micro and macro metastases and were respectively 29.3, 9.3 and $7.9 \%$ when chemotherapy was administered after or before cALND and without chemotherapy.

\section{Background}

The firsts randomized trials confirmed that sentinel lymph node biopsy (SLNB) accurately staged the axilla if sentinel nodes (SN) were not involved [1-3]. If $\mathrm{SN}$ was involved, standard practice was complementary axillary lymph node dissection (cALND) [4]. Axillary recurrences are rare, even with omission of ALND [5, 6]. The main use of axillary surgery is a disease staging procedure and ALND may have a favorable effect on survival [2]. However, the side-effects of ALND including lymphedema, pain and reduced arm movement are higher in comparison with SLNB alone [1, 7-9].

SLNB provide information to guide adjuvant treatments complementary to tumor characteristics and particularly molecular subtypes. However, the entire SN serial section examination eventually with cytokeratin immunostaining resulted in the frequent identification of small SN involvement, isolated tumor cells (ITC) or micro-metastases.

Three randomized trials have concluded at non inferiority of cALND omission for patients with involved SN [9-11]. These results conduct to propose to avoid cALND $[12,13]$ for patients with all criteria reported in these trials. However, we can outline strong limitations of these trials to validate this attitude with a high scientific level.

We designed the SERC trial to compare outcomes in patients with SN involvement treated with ALND or no further treatment to the axilla [14]. The aim of this study was to analyze results of the first 1000 patients included in this trial, mainly for adjuvant treatments and non SN (NSN) involvement at cALND.

\section{Methods}

Study design and patients

SERC trial is a multicenter prospective randomized non-inferiority phase-3 trial comparing no ALND with ALND in patients with breast cancer and metastases in the SN, with a stratification planned between SN macrometastases and ITC or micro-metastases. This study is registered with ClinicalTrials.gov, number NCT01717131. The primary objective is to demonstrate that the hazard ratio (SLNB vs ALND) for disease free survival is significantly lower than the non-inferiority margin set to 1.25 . A total number of 3000 patients with 588 events have been calculated in order to answer with an $85 \%$ power and an error risk of 5\% [14].

The first 1000 patients randomized were recruited from 44 institutions over an accrual period of 41 months from July 2012 to December 2015. Women eligible for registration could be any age $>=18$ years, provided they had no previous or concomitant malignancy, pure ductal carcinoma in situ, previous systemic therapy before SLNB, distant metastases, palpable axillary nodes.

Compared to previous randomized trials, patients with one or more positive $\mathrm{SN}$, multicentric tumours, $<=\mathrm{T} 2$ N0, ITC or micro-metastases or macro-metastases with or without capsular effraction were allowed to participate. Neoadjuvant chemotherapy (NAC) with SLNB before chemotherapy, mastectomy or conservative breast surgery was permitted.

Whole breast irradiation (WBI) was recommended after conservative surgery with boost on tumor basin and post mastectomy radiotherapy (PMRT) was proposed according to guidelines used in each center and start 4 to 8 weeks after surgery or after the end of adjuvant chemotherapy (AC). No specific axillary radiotherapy was delivered and 2 tangential fields were recommended for chest or WBI with a total dose of 50 Grays at ICRU point with 25 fractions of 2 Grays during 5 weeks. AC and 
endocrine-therapy (ET) were proposed according to guidelines used in each center.

The protocol was approved by the institutional review boards of all participating centers, and all participants provided written informed consent. Data were collected from participating centers through electronic CRFs, and centralized at the Institute-Paoli-Calmettes Data-CenterUnit.

\section{Randomization}

The patients were randomly assigned, via a centralized interactive voice-response system (IVRS), to receive $(1,1$ ratio) either ALND or SLNB alone. Randomization was done with a permuted block randomization scheme stratified by participating center and $\mathrm{SN}$ status.

\section{Procedures}

SN detection could be performed by combined isotopic and colorimetric methods or only isotopic method with peri-tumoral or retro-areolar injection. The SN could be examined intra-operatively and ALND done during the operation to remove the primary tumor or post operatively and later second surgery done if randomly assigned to undergo ALND. Axillary ultra-sonography was a systematic recommended pre-operative exam but was not recorded in the trial.

All SN were entirely sectioned at $50-200 \mu \mathrm{m}$ intervals and all sections were examined with hematoxylin and eosin staining (HES) by pathologists at each participating center. Cytokeratin immunostaining was used only when HES was negative. SN could be examined by one step nucleic acid amplification method. A lysate with CK19 mRNA copy number/ $\mu$ l ranging between 250 and 5000 was classified as micro-metastases and greater than 5000 as macro-metastases.

\section{Statistical analysis}

The cut-off date for data collection was May 29, 2016. Of the first 1000 randomized patients, only patients with monitoring review of eligibility data were included in the analysis set. Graphical display of cumulative numbers of accruals since study start, total accruals per participating centers were presented. All patients are grouped according to the treatment they had actually received (ALND or SLNB alone). Descriptive summaries of individual data (age, SBR grade, tumor histology, tumor size, lymphovascular involvement (LVI), SN status, hormonal receptors (HR), tumor sub types) and treatment characteristics (radiotherapy and endocrine therapy (ET)) in the ALND and SLNB groups are presented as mean \pm standard deviation, median (interquartile range) for continuous data, frequency (percent) for categorical data in the full analysis set and in the ALND and SLNB groups. Differences between actually-received treatment groups were evaluated using Mann-Whitney U and Chi-square tests as appropriate. Summary data according to the status of SN were also reported. AC rates were analyzed in the full analysis cohort and in both ALND and SLNB groups. NSN involvement rates were analyzed for patients with ALND in order to determine significant factors correlated with $\mathrm{AC}$ administration and NSN involvement. Univariate associations between patient, tumor, SLNB and treatment characteristics were assessed using logistic regression analysis. Multivariate logistic regression analysis was further used to identify independent factors associated with $\mathrm{AC}$ administration in HR positive patients and NSN involvement in patients treated by radiotherapy and without NAC.

All statistical analyses were carried out using SASSoftware (Release 9.3, SAS-Institute, Inc., Cary, NC). The level of statistical significance was set to 0.05 , with no adjustment for multiplicity.

\section{Results}

\section{Patient accruals and characteristics}

The first 1000 randomized patients were accrued from 44 centers (Fig. 1). The number of patients included in each center ranged between 1 to 262 (Fig. 2). Thirtyseven patients were excluded from the analysis set because of CRF empty (6) or incomplete monitoring (31). Of the 963 patients included in the analysis set, 478 were randomized to receive cALND and 485 SLNB alone. All patient demographics and tumor characteristics were balanced between the two arms (data not shown). Overall, median age was 58 years old $($ C195\% $=57.6-59)$, median tumor size was $18 \mathrm{~mm}(\mathrm{CI} 95 \%=19-20.4)$, median number of harvested SN was $2(689<=2,272>2)$ and median number of involved-SN was $1(925<=2,14>2)$. The status of involved-SN was not determined in 60 patients (6.2\%). SN ITC were present in $6.31 \%$, micro-metastases in $33.0 \%$ and macro-metastases in $60.7 \%$. Of the 846 patients with SN micro or macro-metastases, 289 (34.2\%) were non-eligible to Z0011 criteria: capsular effraction $(n=157)$, mastectomy $(n=145)$, NAC $(n=25),>2$ involved SN $(n=13)$. Of the 355 patients with SN ITC or micrometastases, 11 did not meet the eligibility criteria of IBCSG-23-01: NAC $(n=8),>2$ involved SN $(n=8)$ and tumor size $(\mathrm{n}=1)$.

Forty-nine patients $(4.9 \%)$ did not receive the study treatment as randomized: $42(8.79 \%)$ in the ALND-arm did not have ALND and 7 (1.44\%) in the SLNBalone-arm had ALND with in summary, 443 who received cALND and 520 SLNB-alone. These protocol deviations were in relation with patient's decision in 41 cases $(7$ for ITC SN, 14 micro-metastases, 18 macro-metastases and 2 unknown $\mathrm{SN}$-status) and in relation with investigator's decision in 2 cases (1 ITC, $1 \mathrm{pN} 1 \mathrm{mi}$ ) to avoid cALND in ALND arm, with patient's decision in 1 case (SN macro-metastases), with investigator's decision in 5 cases 


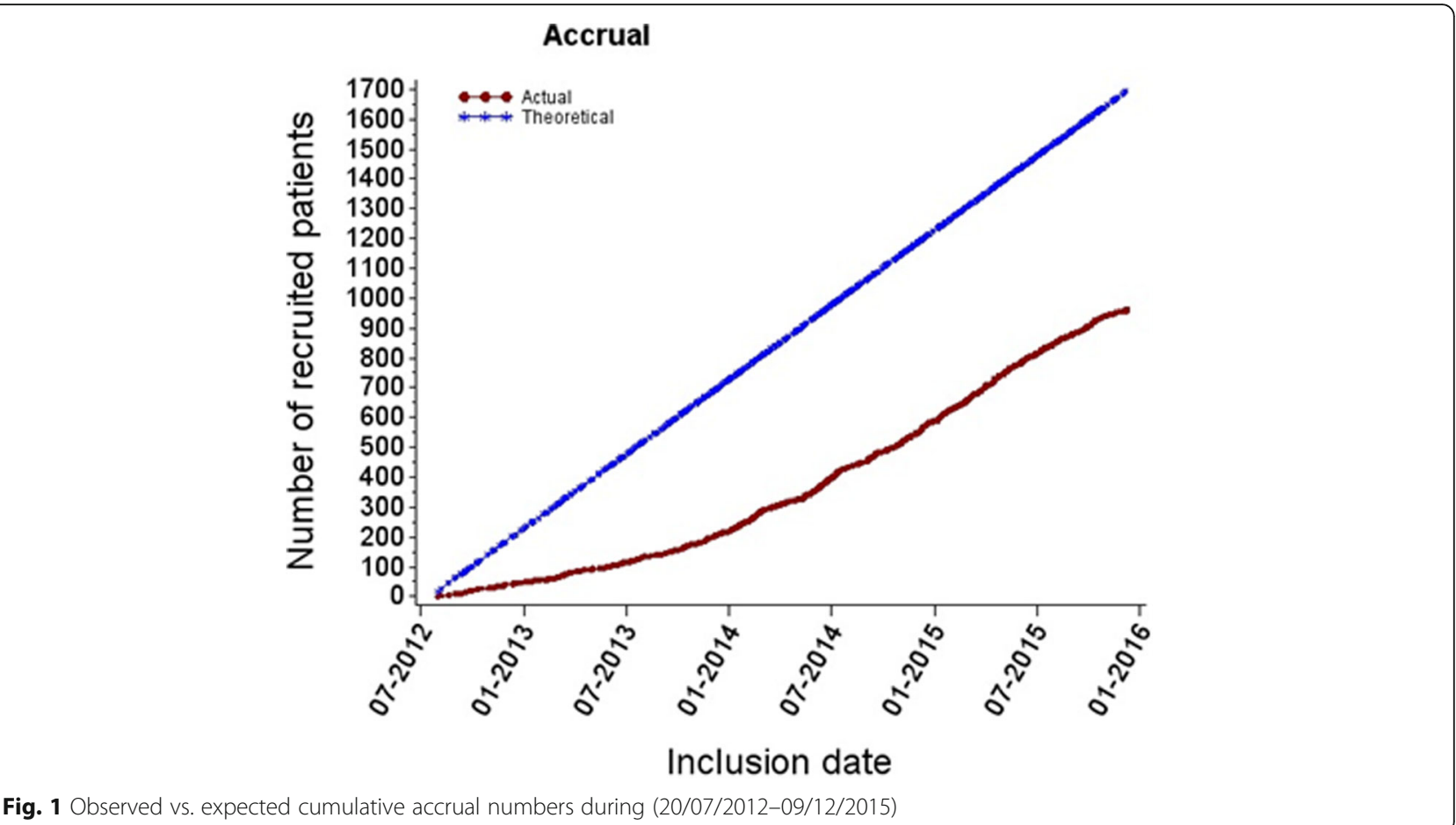

(1 micro-metastases, 4 macro-metastases) and 2 cases without precision (1 SN macro-metastases and 1 unknown SN-status) to performed cALND in SLNB arm. No significant differences in patient's characteristics (age, grade, tumor histology, tumor size, LVI, SN-status, HR and tumor sub types) and adjuvant treatment (AC, radiotherapy, ET, trastuzumab and type of surgery) were observed between the two actual treatment groups (Tables 1 and 2).

\section{Adjuvant treatments}

Full treatment information was not available in patients who did not complete the full sequence of treatment at the date of last follow-up for this analysis. Whole breast irradiation or PMRT was delivered in $95.9 \%$ of patients, including 134 PMRT (82.7\%: 134/162): 94.8\% (92/97) for macro-metastatic SN and 61.8\% (34/55) for ITC or micrometastases. PMRT rate was not significantly different

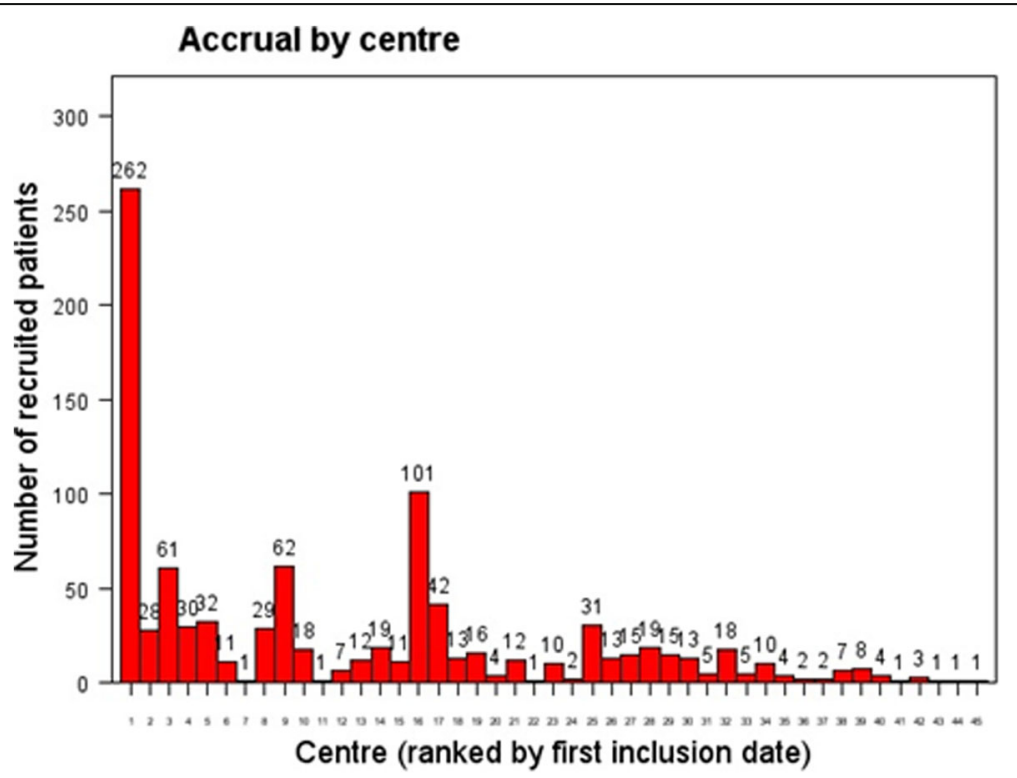

Fig. 2 Number of patients accrued by participating center during the accrual period of first 1000 randomized patients: The majority of patients (528) were recruited from 4 different institutions and 20 centers accrued less than 10 patients 
Table 1 Baseline patient, tumor and prior surgery treatment characteristics according to actually received study group (ALND vs SLNB)

\begin{tabular}{|c|c|c|c|c|c|}
\hline Test & Class & All $(n=963)$ & $\operatorname{ALND}(n=443)$ & SLNB alone $(n=520)$ & $\overline{p \text {-value }}$ \\
\hline \multirow[t]{4}{*}{$\overline{\text { Age }}$} & Median [range] & $58[26-84]$ & 59 [33-84] & $58[26-84]$ & 0.761 \\
\hline & $<=40$ & $51(5.30)$ & $26(5.87)$ & $25(4.81)$ & 0.7142 \\
\hline & $41-75$ & 858 (89.10) & 391 (88.26) & $467(89.81)$ & \\
\hline & $>75$ & $54(5.61)$ & $26(5.87)$ & $28(5.38)$ & \\
\hline \multirow[t]{5}{*}{ Clinical T stage } & $\mathrm{T} 1$ & $571(59.60)$ & 261(59.32) & $310(59.85)$ & 0.9393 \\
\hline & $\mathrm{T} 2$ & $342(35.70)$ & $156(35.45)$ & $186(35.91)$ & \\
\hline & $\mathrm{T} 3$ & $17(1.77)$ & $9(2.05)$ & $8(1.54)$ & \\
\hline & $\mathrm{T} 4$ & $5(0.52)$ & $2(0.45)$ & $3(0.58)$ & \\
\hline & $\mathrm{Tis} / \mathrm{Tx}$ & $23(2.40)$ & $12(2.73)$ & $11(2.12)$ & \\
\hline \multirow[t]{4}{*}{ Tumor size (mm) } & Median [range] & 18 [0-81] & $18[0-80]$ & 18 [0-81] & 0.573 \\
\hline & $<=10$ & $151(15.83)$ & $64(14.61)$ & $87(16.86)$ & 0.5097 \\
\hline & $10-30$ & $704(73.79)$ & $331(75.57)$ & $373(72.29)$ & \\
\hline & $>30$ & $99(10.38)$ & $43(9.82)$ & $56(10.85)$ & \\
\hline \multirow[t]{4}{*}{ Histology Type } & Infiltrating ductal & $781(81.10)$ & 358(80.81) & 423(81.35) & 0.8805 \\
\hline & Infiltrating lobular & $102(10.59)$ & $45(10.16)$ & $57(10.96)$ & \\
\hline & Mixed & $26(2.70)$ & $13(2.93)$ & $13(2.50)$ & \\
\hline & Other & $54(5.61)$ & $27(6.09)$ & $27(5.19)$ & \\
\hline \multirow[t]{3}{*}{ SBR Grade } & Gr I & $213(22.76)$ & $101(23.60)$ & $112(22.05)$ & 0.2677 \\
\hline & Gr $\|$ & $479(51.18)$ & 207 (48.36) & $272(53.54)$ & \\
\hline & Gr III & $244(26.07)$ & $120(28.04)$ & $124(24.41)$ & \\
\hline \multirow[t]{2}{*}{ LVI } & No & $281(30.38)$ & $134(31.60)$ & $147(29.34)$ & 0.4560 \\
\hline & Yes & $644(69.62)$ & $290(68.40)$ & $354(70.66)$ & \\
\hline \multirow[t]{2}{*}{ Capsular Effraction } & No & $698(80.14)$ & $327(80.74)$ & $371(79.61)$ & 0.6775 \\
\hline & Yes & $173(19.86)$ & 78 (19.26) & 95 (20.39) & \\
\hline \multirow[t]{2}{*}{ Hormonal receptors status } & Negative & $84(8.89)$ & $44(10.16)$ & $40(7.81)$ & 0.2061 \\
\hline & Positive & $861(91.11)$ & $389(89.84)$ & $472(92.19)$ & \\
\hline \multirow[t]{2}{*}{ HER2 status } & Negative & $815(87.73)$ & 369 (86.42) & $446(88.84)$ & 0.26106 \\
\hline & Positive & $114(12.27)$ & $58(13.58)$ & $56(11.16)$ & \\
\hline \multirow[t]{4}{*}{ Tumor subtype (RH/HER2) } & $\mathrm{RH}+/ \mathrm{HER} 2+$ & $83(9.00)$ & $41(9.72)$ & $42(8.40)$ & 0.5150 \\
\hline & $\mathrm{RH}+/ \mathrm{HER} 2-$ & $759(82.32)$ & 339 (80.33) & $420(84.00)$ & \\
\hline & RH-/HER2+ & $30(3.25)$ & $16(3.79)$ & $14(2.80)$ & \\
\hline & RH-/HER2- & $50(5.42)$ & $26(6.16)$ & $24(4.80)$ & \\
\hline \multirow[t]{5}{*}{ Harvested SN, number } & Median [range] & $2[0-8]$ & $2[0-7]$ & $2[1-8]$ & 0.996 \\
\hline & 0 & $2(0.21)$ & $2(0.45)$ & & 0.2823 \\
\hline & 1 & $373(38.73)$ & 168 (37.92) & $205(39.42)$ & \\
\hline & $\geq 2$ & $588(61.06)$ & $273(61.63)$ & $315(60.58)$ & \\
\hline & Median [range] & $1[0-4]$ & $1[0-4]$ & $1[0-3]$ & 0.422 \\
\hline \multirow[t]{3}{*}{ Involved SN } & $<=2$ & $925(98.51)$ & $424(98.60)$ & $501(98.43)$ & 0.8242 \\
\hline & $>2$ & 14 (1.49) & $6(1.40)$ & $8(1.57)$ & \\
\hline & ITC & $57(6.31)$ & $22(5.30)$ & 35 (7.17) & 0.5040 \\
\hline \multirow[t]{2}{*}{ SN status } & Micro & $298(33.00)$ & 137 (33.01) & 161 (32.99) & \\
\hline & Macro & $548(60.69)$ & 256 (61.69) & $292(59.84)$ & \\
\hline
\end{tabular}

$P$ value based on Wilcoxon rank sum test for continuous variables or Chi square test for categorical variables 
Table 2 Surgical and adjuvant treatment characteristics according to actually received study group (ALND vs SLNB)

\begin{tabular}{|c|c|c|c|c|c|}
\hline Test & Classes & All $(n=963)$ & $\operatorname{ALND}(n=443)$ & SLNB $(n=520)$ & $p$-value \\
\hline \multirow[t]{2}{*}{ Breast surgery Type } & Mastectomy & $170(17.65)$ & $77(17.38)$ & $93(17.88)$ & 0.8383 \\
\hline & Conservative & $793(82.35)$ & $366(82.62)$ & $427(82.12)$ & \\
\hline \multirow[t]{2}{*}{ Radiotherapy } & No & $38(4.07)$ & $19(4.45)$ & 19 (3.75) & 0.5885 \\
\hline & Yes & 896 (95.93) & $408(95.55)$ & $488(96.25)$ & \\
\hline \multirow[t]{3}{*}{ Chemotherapy } & No & $282(29.59)$ & $126(28.70)$ & $156(30.35)$ & 0.3482 \\
\hline & Neoadjuvant & $27(2.83)$ & $16(3.64)$ & $11(2.14)$ & \\
\hline & Adjuvant & $644(67.58)$ & $297(67.65)$ & $347(67.51)$ & \\
\hline \multirow[t]{2}{*}{ Endocrine therapy } & No & 78 (10.39) & $30(8.72)$ & $48(11.79)$ & 0.1691 \\
\hline & Yes & $673(89.61)$ & $314(91.28)$ & $359(88.21)$ & \\
\hline \multirow[t]{2}{*}{ Trastuzumab } & No & $578(85.76)$ & $261(85.02)$ & 317 (86.38) & 0.6149 \\
\hline & Yes & $96(14.24)$ & $46(14.98)$ & $50(13.62)$ & \\
\hline
\end{tabular}

$P$ value based on Wilcoxon rank sum test for continuous variables or Chi square test for categorical variables

between two arms: $82.7 \%(72 / 87)$ without ALND and $82.7 \%(62 / 75)$ with ALND. AC was administered in $69.5 \%$ of patients $(644 / 926)$, ET in $89.6 \%$ and the proportions were similar in the two actual treatment groups: 237 of $423(70.2 \%)$ in the ALND group and 347 of $503(69.0 \%)$ in the SLNB alone received AC, 314 of $344(91.3 \%)$ in the ALND group and 359 of 407 (88.2\%) in the SLNB alone received ET. Patients, tumor and treatment characteristics (age, grade, tumor histology, tumor size, LVI, SN status, HR, tumor sub types, ET and radiotherapy) were significantly different in univariate analysis according to chemotherapy administration or not, without evidence of any difference in crudes rates of $\mathrm{AC}$ between ALND and SLNB groups (Table 3).

$\mathrm{AC}$ was administered in $97 \%$ for patients with $\mathrm{HR}$ - tumors (73/75), 67.7\% for patients with HR+ tumors (568/837), $55.4 \%$ for patients with SN involved by ITC (31/56), 56.8\% for SN micro-metastases (164/289), $77.1 \%$ for SN macrometastases (405/525). Among $\mathrm{HR}+$ patients, multivariate analysis identified age, tumor size, LVI, HER2 status, grade and SN status as significant predictors of AC. Chemotherapy use increased for: tumors with size $11-30 \mathrm{~mm}(\mathrm{OR}=2.72)$ or $>30 \mathrm{~mm}$ (OR: 2.65), ductal carcinoma $(\mathrm{OR}=2.10)$, tumors with LVI $(\mathrm{OR}=2.16)$, SBR grade $2(\mathrm{OR}=3.05)$ or grade $3(\mathrm{OR}=25.95)$, HER2+ $(\mathrm{OR}=3.12)$, SN macrometastases $(\mathrm{OR}=3.70)$, and for women age between 41 and 75 years old $(\mathrm{OR}=13.9)$ or $<=40$ years $(\mathrm{OR}=75.8)$ (Table 4). Forty patients had involved $\mathrm{SN}>2$ : all presented $\mathrm{HR}+$ tumors and one did not received AC.

\section{Final pathological findings in ALND group}

Of the 443 patients who underwent ALND, the number of involved NSN was reported missing in one patient. The overall rate of positive NSN was $19 \%$ for patients with cALND. Crude rates of positive NSN according to SN status were $4.5 \%$ for patients with ITC (1/22), $9.5 \%$ for SN micro-metastases (13/137), 23.9\% for SN macro- metastases (61/255). Univariate analysis of patient and tumor characteristics revealed that positive NSN rates were significantly higher for patients with tumor sizes $>10 \mathrm{~mm},>2$ involved SN, macro-metastatic SN, presence of LVI and SN capsular effraction (Table 5). Crude rates of positive NSN rates were significantly higher in patients who received radiotherapy or chemotherapy. Of the 19 patients in the ALND group who did not received radiotherapy, none reported positive NSN. Crude rates of positive NSN were $7.9 \%$ for patients without systemic therapy (13/142), $18.8 \%$ with NAC (3/16) and $23.9 \%$ with AC (71/297).

Systemic therapy was administered in $88.1 \%$ for patients with involved NSN (74/84) of whom $11.9 \%$ with chemotherapy first administrated prior to ALND (10/84) and $76.2 \%$ with chemotherapy administered after ALND (64/84). As ALND omission precludes the observation of positive NSN, logistic regression was used to identify non determinant factors of AC predictive of NSN involvement. In addition to patients who received NAC, patients who did not receive radiotherapy were excluded due the lack of positive NSN. Multivariate regression analysis taking into account key determinant factors of AC identified capsular effraction $(\mathrm{OR}=2.31, p=0.028)$ and involved $\mathrm{SN}>2(\mathrm{OR}=8.16, p=0.081)$ as significant or of borderline significance predictors of NSN involvement (Table 6). Multivariate analysis excluding patients who received chemotherapy prior to ALND lead to the same conclusions (capsular effraction: $\mathrm{OR}=2.36, p=0.038$; involved $\mathrm{SN}>2: \mathrm{OR}=6.78, p=0.12$ ) (Table 7).

For patients with cALND $(n=419)$, involved NSN rates were respectively $29.36 \%$ (64/218) when chemotherapy was administered after cALND, 9.33\% (7/75) when chemotherapy was administered before cALND and 7.94\% (10/126) for patients without AC. For SN ITC, involved NSN rate was $4.5 \%(1 / 22)$ with chemotherapy administered after ALND. Involved NSN rates were respectively 
Table 3 Baseline patient, tumor and treatment characteristics according to adjuvant chemotherapy administration

\begin{tabular}{|c|c|c|c|c|c|c|c|c|c|c|}
\hline \multirow[t]{2}{*}{ Test } & \multirow[b]{2}{*}{ Category } & \multicolumn{3}{|l|}{ All } & \multicolumn{2}{|c|}{ ALND } & \multicolumn{2}{|c|}{ SLNB } & \multicolumn{2}{|l|}{ SLBN vs ALND } \\
\hline & & $\bar{N}$ & $n(\%)$ & $p$ value $^{*}$ & $\bar{N}$ & $n(\%)$ & $\bar{N}$ & $n(\%)$ & Odd Ratio95\%Cl & $p$ value ${ }^{* *}$ \\
\hline \multirow[t]{3}{*}{ Age in class } & $<=40$ & 45 & $44(.98)$ & $<.001$ & 22 & $21(.95)$ & 23 & $23(1.0)$ & $>999[<0.01,>999]$ & 0.978 \\
\hline & $41-75$ & 828 & $582(.70)$ & & 376 & 266(.71) & 452 & $316(.70)$ & $0.96[0.71,1.30]$ & 0.794 \\
\hline & $>75$ & 53 & $18(.34)$ & & 25 & $10(.40)$ & 28 & $8(.29)$ & $0.61[0.19,1.88]$ & 0.387 \\
\hline \multirow[t]{3}{*}{ Tumor size (mm) } & $<=10$ & 143 & $64(.45)$ & & 57 & $27(.47)$ & 86 & $37(.43)$ & $0.84[0.43,1.64]$ & 0.610 \\
\hline & $10-30$ & 684 & $506(.74)$ & & 323 & 236(.73) & 361 & $270(.75)$ & $1.09[0.78,1.54]$ & 0.608 \\
\hline & $>30$ & 96 & $73(.76)$ & $<.001$ & 41 & $34(.83)$ & 55 & $39(.71)$ & $0.51[0.19,1.37]$ & 0.179 \\
\hline \multirow[t]{4}{*}{ Histology type } & Infiltrant ductal & 748 & $543(.73)$ & & 340 & 246(.72) & 408 & 297(.73) & $1.02[0.74,1.41]$ & 0.893 \\
\hline & Infiltrant lobular & 101 & $50(.50)$ & & 45 & 25(.56) & 56 & $25(.45)$ & $0.65[0.30,1.42]$ & 0.279 \\
\hline & Mixed & 25 & $19(.76)$ & $<.001$ & 12 & $10(.83)$ & 13 & $9(.69)$ & $0.46[0.07,3.05]$ & 0.425 \\
\hline & Other & 52 & $32(.62)$ & & 26 & $16(.62)$ & 26 & $16(.62)$ & $1.00[0.33,3.02]$ & 1.000 \\
\hline \multirow[t]{3}{*}{ SBR grade } & Gr 1 & 206 & $88(.43)$ & $<.001$ & 98 & $40(.41)$ & 108 & $48(.44)$ & $1.16[0.67,2.01]$ & 0.600 \\
\hline & Gr $\|$ & 464 & $317(.68)$ & & 200 & 139(.70) & 264 & $178(.67)$ & $0.91[0.61,1.35]$ & 0.635 \\
\hline & Gr III & 237 & 223(.94) & & 116 & $110(.95)$ & 121 & 113(.93) & $0.77[0.26,2.29]$ & 0.640 \\
\hline \multirow[t]{2}{*}{ LVI } & No & 275 & $235(.85)$ & $<.001$ & 130 & $110(.85)$ & 145 & $125(.86)$ & $1.14[0.58,2.22]$ & 0.709 \\
\hline & Yes & 616 & 394(.64) & & 276 & $178(.64)$ & 340 & $216(.64)$ & $0.96[0.69,1.34]$ & 0.805 \\
\hline \multirow[t]{2}{*}{ Capsular Effraction } & No & 673 & 467(.69) & & 313 & 217(.69) & 360 & $250(.69)$ & $1.01[0.72,1.40]$ & 0.974 \\
\hline & Yes & 167 & $122(.73)$ & 0.355 & 75 & $56(.75)$ & 92 & $66(.72)$ & $0.86[0.43,1.72]$ & 0.673 \\
\hline \multirow[t]{2}{*}{ Hormonal receptors status } & Negative & 75 & $73(.97)$ & $<.001$ & 39 & $38(.97)$ & 36 & $35(.97)$ & $0.92[0.06,15.02]$ & 0.955 \\
\hline & Positive & 837 & $568(.68)$ & & 377 & $257(.68)$ & 460 & $311(.68)$ & $0.97[0.73,1.30]$ & 0.863 \\
\hline \multirow[t]{2}{*}{ HER2 status } & Negative & 794 & $532(.67)$ & & 358 & $242(.68)$ & 436 & $290(.67)$ & $0.95[0.71,1.28]$ & 0.747 \\
\hline & Positive & 102 & $98(.96)$ & $<.001$ & 52 & $50(.96)$ & 50 & $48(.96)$ & $0.96[0.13,7.02]$ & 0.968 \\
\hline \multirow[t]{4}{*}{ Tumor subtype (RH/HER2) } & $\mathrm{RH}+/ \mathrm{HER} 2+$ & 75 & $72(.96)$ & & 36 & $35(.97)$ & 39 & $37(.95)$ & $0.53[0.05,6.05]$ & 0.612 \\
\hline & $\mathrm{RH}+/ \mathrm{HER} 2-$ & 743 & $486(.65)$ & & 332 & $218(.66)$ & 411 & $268(.65)$ & $0.98[0.72,1.33]$ & 0.897 \\
\hline & RH-/HER2+ & 26 & $26(1.0)$ & $<.001$ & 15 & $15(1.0)$ & 11 & $11(1.0)$ & & \\
\hline & RH-/HER2- & 45 & $44(.98)$ & & 22 & $22(1.0)$ & 23 & $22(.96)$ & $<0.01[<0.01,>999.99]$ & 0.978 \\
\hline \multirow[t]{3}{*}{ Harvested SN, number } & $=1$ & 365 & 253(.69) & & 165 & 115(.70) & 200 & 138(.69) & $0.97[0.62,1.51]$ & 0.886 \\
\hline & $=2$ & 303 & 205(.68) & & 142 & $96(.68)$ & 161 & 109(.68) & $1.00[0.62,1.63]$ & 0.986 \\
\hline & $>2$ & 258 & $186(.72)$ & 0.519 & 116 & $86(.74)$ & 142 & $100(.70)$ & $0.83[0.48,1.44]$ & 0.509 \\
\hline \multirow[t]{2}{*}{ Involved SN } & $<=2$ & 891 & 619(.69) & & 406 & 285(.70) & 485 & $334(.69)$ & $0.94[0.71,1.25]$ & 0.668 \\
\hline & $>2$ & 14 & $13(.93)$ & 0.059 & 6 & $6(1.0)$ & 8 & $7(.88)$ & $<0.01[<0.01,>999.99]$ & 0.979 \\
\hline \multirow[t]{3}{*}{ SN status } & ITC & 56 & $31(.55)$ & $<.001$ & 22 & 14(.64) & 34 & $17(.50)$ & $0.58[0.19,1.71]$ & 0.322 \\
\hline & Micro & 289 & $164(.57)$ & & 133 & $76(.57)$ & 156 & $88(.56)$ & $0.97[0.61,1.55]$ & 0.900 \\
\hline & Macro & 525 & $405(.77)$ & & 241 & 183(.76) & 284 & $222(.78)$ & $1.13[0.75,1.71]$ & 0.544 \\
\hline \multirow[t]{2}{*}{ Endocrine therapy } & No & 75 & $66(.88)$ & $<.001$ & 29 & $28(.97)$ & 46 & $38(.83)$ & $0.17[0.02,1.45]$ & 0.106 \\
\hline & Yes & 653 & $420(.64)$ & & 302 & 192(.64) & 351 & $228(.65)$ & $1.06[0.77,1.46]$ & 0.714 \\
\hline \multirow[t]{2}{*}{ Trastuzumab } & No & 567 & $368(.65)$ & & 256 & $162(.63)$ & 311 & $206(.66)$ & $1.14[0.81,1.61]$ & 0.463 \\
\hline & Yes & 83 & $83(1.0)$ & $<.001$ & 38 & $38(1.0)$ & 45 & $45(1.0)$ & & \\
\hline \multirow[t]{2}{*}{ Breast Surgery } & Mastectomy & 160 & $120(.75)$ & 0.099 & 73 & $55(.75)$ & 87 & $65(.75)$ & $0.97[0.47,1.98]$ & 0.927 \\
\hline & Conservative & 766 & $524(.68)$ & & 350 & 242(.69) & 416 & $282(.68)$ & $0.94[0.69,1.28]$ & 0.688 \\
\hline \multirow[t]{2}{*}{ Radiotherapy } & No & 38 & $19(.50)$ & 0.010 & 19 & $10(.53)$ & 19 & $9(.47)$ & $0.81[0.23,2.86]$ & 0.749 \\
\hline & Yes & 867 & $604(.70)$ & & 390 & 273(.70) & 477 & $331(.69)$ & $0.97[0.73,1.30]$ & 0.846 \\
\hline
\end{tabular}


Table 4 Multivariate analysis of factors associated with chemotherapy administration in hormone-receptor positive patients

\begin{tabular}{|c|c|c|c|c|c|}
\hline Effect & $P$ value & Contrast & Odd Ratio Estimate & $95 \% \mathrm{Cl}$ & $P$ value \\
\hline \multirow[t]{2}{*}{ Age in class } & $<.0001$ & $41-75$ vs $>75$ & 13.894 & {$[5.050 ; 38.228]$} & $<.0001$ \\
\hline & & $<=40$ vs $>75$ & 75.801 & {$[14.803 ; 388.145]$} & $<.0001$ \\
\hline \multirow[t]{2}{*}{ Tumor Size } & 0.0017 & $10-30$ vs $<=10$ & 2.724 & {$[1.567 ; 4.737]$} & 0.0004 \\
\hline & & $>30$ vs $<=10$ & 2.654 & {$[1.060 ; 6.646]$} & 0.0372 \\
\hline LVI & 0.0036 & Pos. Vs Neg. & 2.163 & {$[1.287 ; 3.634]$} & 0.0036 \\
\hline HER2 Status & 0.0470 & Pos. Vs Neg. & 3.120 & {$[1.015 ; 9.588]$} & 0.0470 \\
\hline \multirow[t]{2}{*}{ Histology Type } & 0.0930 & Mixed/Other vs lobular & 1.866 & {$[0.716 ; 4.863]$} & 0.2020 \\
\hline & & Ductal vs lobular & 2.096 & {$[1.077 ; 4.077]$} & 0.0293 \\
\hline \multirow[t]{2}{*}{ SBR Grade } & $<.0001$ & Gr II vs Gr I & 3.052 & {$[1.875 ; 4.967]$} & $<.0001$ \\
\hline & & Gr III vs Gr I & 25.946 & {$[10.448 ; 64.435]$} & $<.0001$ \\
\hline $\mathrm{Nb}$ Involved SN & 0.4193 & $>2$ vs $<=2$ & 2.635 & {$[0.251 ; 27.672]$} & 0.4193 \\
\hline \multirow[t]{2}{*}{ SN Status } & $<.0001$ & Macro vs ITC & 3.695 & {$[1.571 ; 8.692]$} & 0.0027 \\
\hline & & Micro vs ITC & 1.447 & {$[0.603 ; 3.474]$} & 0.4078 \\
\hline Surgery & 0.2921 & Conservative vs Mastectomy & 0.715 & {$[0.382 ; 1.335]$} & 0.2921 \\
\hline Hormonotherapy & 0.6144 & Yes vs No & 1.429 & {$[0.357 ; 5.721]$} & 0.6144 \\
\hline Radiotherapy & 0.3787 & Yes vs No & 1.636 & {$[0.547 ; 4.899]$} & 0.3787 \\
\hline ALND status & 0.5602 & Yes vs No & 0.881 & {$[0.574 ; 1.350]$} & 0.5602 \\
\hline
\end{tabular}

Abbreviations: $A L N D$ axilliary lymph node dissection, SLNB sentinel lymph node dissection alone, $C I$ confidence interval

$5.3 \%$ (3/57) without chemotherapy, $0 \% \quad(0 / 17)$ with chemotherapy administered before ALND, 21.7\% (10/46) with chemotherapy administered after ALND for SN micro-metastases and respectively $12.0 \%$ (7/58), 15.5\% (7/ $45)$ and $51.8 \%(44 / 85)$ for SN macro-metastases.

We reported only one positive NSN in 49 patients (58.3\%), 2 positive NSN in $9(10.7 \%)$ and 3 or more in $26(31.0 \%)$, respectively $64.3 \%(9 / 14), 21.4 \%(3 / 14)$ and $14.3 \%(2 / 14)$ for involved $\mathrm{SN}$ by ITC or micrometastases and 55.7\% (34/61), 6.6\% (4/61) and 37.7 (23/61) for SN macro-metastases. Number of positive NSN according to administration time of $\mathrm{AC}$ were respectively for no chemotherapy, ALND before chemotherapy and ALND after chemotherapy: only one positive NSN in $60 \%$ (6/10), 54.7\% (35/64) and 71.4\% (5/7) patients, 2 positive NSN in $20 \%(2 / 10), 9.4 \%(6 / 64)$ and $14.3 \%(1 / 7)$ patients and $\geq 3$ positive NSN in $20 \%(2 / 10), 35.9 \%(23 / 64)$ and $14.3 \%(1 / 7)$ patients.

For 256 patients non eligible to Z0011 with SN macrometastases in all patients, 33 (30.3\%: 33/109) had involved NSN, respectively 19,3 and 11 with 1,2 and $\geq 3$ positive NSN. When cALND was performed after chemotherapy, positive NSN rate was $19.4 \%(6 / 31)$ in comparison with $48.1 \%(25 / 52)$ for patients with cALND performed before chemotherapy and $8.0 \%(2 / 25)$ with cALND without chemotherapy $(p<0.001)$. There was no difference between ALND and no ALND, respectively 109 and 116 patients (48.4 and 51.6\%). Chemotherapy was delivered in $92.4 \%$ of these patients, ET in $91.5 \%$, trastuzumab in
$13.8 \%$, radiotherapy in $96.8 \%$ and surgery was a total mastectomy in $44.9 \%$ with PMRT in $93.9 \%$.

\section{Discussion}

In this study 963 patients were evaluable and actually 1834 patients were included in SERC trial. Number of included patients was respectively 856, 931 and 233 in ACOSOG-Z0011 [10], IBCSG-23-01 [9] and AATRM trials [11], respectively from 177 institutions between May 2001 and December 2014, 27 institutions between April 2001 and February 2010, 18 institutions between January 2001 and December 2008. However, in Z0011 and IBCSG-23-01 less than 50\% of patients were included in comparison with initial effective calculated to be able to demonstrate equivalence between the two arms. In AATRM trial, the sample size was estimated to be 352 patients and a maximum difference of $15 \%$ in disease free survival for the experimental group was established as clinically significant.

In SERC trial, about 287 patients were included per year and actually with 80 institutions about 340 patients per year are included. In comparison, for Z0011, IBCSG23-01 and AATRM, numbers of patients included per year were respectively 63, 103 and 33 .

In our study, 49 patients (4.9\%) did not received the study treatment as randomized: 42 in the ALND arm did not have ALND (8.79\%), and 7 in the SLND-alone arm had ALND (1.44\%). Similar results were reported in Z0011 trial: 43 patients (5.0\%) did not receive the study treatment as randomized: 32 in the ALND group (7.6\%) 
Table 5 Baseline patient, tumor and prior surgery treatment characteristics according to non sentinel Node (NSN) involvement

\begin{tabular}{|c|c|c|c|c|c|}
\hline \multirow[t]{2}{*}{ Test } & \multirow[b]{2}{*}{ Class } & \multirow[b]{2}{*}{ All $(n=443)$} & \multicolumn{3}{|c|}{ Non sentinel node involvment } \\
\hline & & & No $(n=358)$ & Yes $(n=84)$ & $p$-value \\
\hline \multirow[t]{4}{*}{ Age } & Median [range] & $59[33-84]$ & 59 [33-84] & 59 [39-84] & 0.828 \\
\hline & $<=40$ & $26(5.87)$ & $24(6.70)$ & $2(2.38)$ & 0.2864 \\
\hline & $41-75$ & $391(88.26)$ & $314(87.71)$ & $76(90.48)$ & \\
\hline & $>75$ & $26(5.87)$ & $20(5.59)$ & $6(7.14)$ & \\
\hline \multirow[t]{5}{*}{ Clinical T stage } & $\mathrm{T} 1$ & $261(59.32)$ & $216(60.67)$ & $44(53.01)$ & 0.2384 \\
\hline & $\mathrm{T} 2$ & $156(35.45)$ & $123(34.55)$ & $33(39.76)$ & \\
\hline & $\mathrm{T} 3$ & $9(2.05)$ & $5(1.40)$ & $4(4.82)$ & \\
\hline & $\mathrm{T} 4$ & $2(0.45)$ & $2(0.56)$ & & \\
\hline & $\mathrm{Tis} / \mathrm{Tx}$ & $12(2.73)$ & $10(2.81)$ & $2(2.41)$ & \\
\hline \multirow[t]{4}{*}{ Tumor size (mm) } & Median [range] & $18[0-80]$ & 18 [0-70] & 19 [0-80] & 0.059 \\
\hline & $<=10$ & $64(14.61)$ & $55(15.58)$ & $9(10.71)$ & 0.0465 \\
\hline & $10-30$ & $331(75.57)$ & $269(76.20)$ & $61(72.62)$ & \\
\hline & $>30$ & $43(9.82)$ & $29(8.22)$ & $14(16.67)$ & \\
\hline \multirow[t]{4}{*}{ Histology Type } & Infiltrating ductal & $358(80.81)$ & $290(81.01)$ & $67(79.76)$ & 0.4021 \\
\hline & Infiltrating lobular & $45(10.16)$ & $33(9.22)$ & $12(14.29)$ & \\
\hline & Mixed & $13(2.93)$ & $11(3.07)$ & $2(2.38)$ & \\
\hline & Other & $27(6.09)$ & $24(6.70)$ & $3(3.57)$ & \\
\hline \multirow[t]{3}{*}{ SBR Grade } & Gr I & $101(23.60)$ & $88(25.51)$ & $12(14.63)$ & 0.1104 \\
\hline & Gr $\|$ & $207(48.36)$ & $162(46.96)$ & $45(54.88)$ & \\
\hline & Gr II & $120(28.04)$ & $95(27.54)$ & $25(30.49)$ & \\
\hline \multirow[t]{2}{*}{ LVI } & No & $134(31.60)$ & $100(29.41)$ & $34(40.96)$ & 0.0425 \\
\hline & Yes & $290(68.40)$ & $240(70.59)$ & $49(59.04)$ & \\
\hline \multirow[t]{2}{*}{ Capsular Effraction } & No & $327(80.74)$ & $276(83.64)$ & $50(67.57)$ & 0.0016 \\
\hline & Yes & $78(19.26)$ & $54(16.36)$ & $24(32.43)$ & \\
\hline \multirow[t]{2}{*}{ Hormonal receptors } & Negative & $44(10.16)$ & $36(10.32)$ & $8(9.64)$ & 0.8546 \\
\hline & Positive & 389 (89.84) & $313(89.68)$ & 75 (90.36) & \\
\hline \multirow[t]{2}{*}{ HER2 status } & Negative & $369(86.42)$ & $297(86.34)$ & 71 (86.59) & 0.9530 \\
\hline & Positive & $58(13.58)$ & $47(13.66)$ & $11(13.41)$ & \\
\hline \multirow[t]{4}{*}{ Tumor subtype (RH/HER2) } & $\mathrm{RH}+/ \mathrm{HER} 2+$ & $41(9.72)$ & $34(10.00)$ & $7(8.64)$ & 0.8656 \\
\hline & $\mathrm{RH}+/ \mathrm{HER} 2-$ & 339 (80.33) & $272(80.00)$ & $66(81.48)$ & \\
\hline & $\mathrm{RH}-/ \mathrm{HER} 2+$ & $16(3.79)$ & $12(3.53)$ & $4(4.94)$ & \\
\hline & RH-/HER2- & $26(6.16)$ & $22(6.47)$ & $4(4.94)$ & \\
\hline \multirow[t]{4}{*}{ Harvested SN, number } & Median [range] & $2[0-7]$ & $2[0-7]$ & $2[0-5]$ & 0.650 \\
\hline & 0 & $2(0.45)$ & $1(0.28)$ & $1(1.19)$ & 0.4885 \\
\hline & 1 & 168 (37.92) & 138 (38.55) & $30(35.71)$ & \\
\hline & $\geq 2$ & $273(61.63)$ & $219(61.17)$ & $53(63.10)$ & \\
\hline \multirow[t]{4}{*}{ Involved SN } & Median [range] & $1[0-4]$ & $1[0-4]$ & $1[0-4]$ & 0.004 \\
\hline & $<=2$ & $424(98.60)$ & $346(99.43)$ & $77(95.06)$ & 0.0026 \\
\hline & $>2$ & $6(1.40)$ & $2(0.57)$ & $4(4.94)$ & \\
\hline & ITC & $22(5.30)$ & $21(6.19)$ & $1(1.33)$ & 0.0005 \\
\hline \multirow[t]{3}{*}{ SN status } & Micro & $137(33.01)$ & $124(36.58)$ & $13(17.33)$ & \\
\hline & Macro & $256(61.69)$ & $194(57.23)$ & $61(81.33)$ & \\
\hline & Mastectomie & 77 (17.38) & 60 (16.76) & $17(20.24)$ & 0.4494 \\
\hline
\end{tabular}


Table 5 Baseline patient, tumor and prior surgery treatment characteristics according to non sentinel Node (NSN) involvement (Continued)

\begin{tabular}{|c|c|c|c|c|c|}
\hline \multirow[t]{2}{*}{ Test } & \multirow[b]{2}{*}{ Class } & \multirow[b]{2}{*}{ All $(n=443)$} & \multicolumn{3}{|c|}{ Non sentinel node involvment } \\
\hline & & & No $(n=358)$ & Yes $(n=84)$ & $p$-value \\
\hline \multirow[t]{2}{*}{ Breast surgery Type } & Conservative & $366(82.62)$ & $298(83.24)$ & $67(79.76)$ & \\
\hline & No & $19(4.45)$ & $19(5.51)$ & & 0.0297 \\
\hline \multirow[t]{2}{*}{ Radiotherapy } & Yes & $408(95.55)$ & $326(94.49)$ & $82(100.0)$ & \\
\hline & No & $126(28.70)$ & $116(32.68)$ & $10(11.90)$ & 0.0007 \\
\hline \multirow[t]{3}{*}{ Chemotherapy } & Neoadjuvant & $16(3.64)$ & $13(3.66)$ & $3(3.57)$ & \\
\hline & Adjuvant & $297(67.65)$ & $226(63.66)$ & $71(84.52)$ & \\
\hline & No & $30(8.72)$ & $25(9.06)$ & $5(7.35)$ & 0.6553 \\
\hline \multirow[t]{2}{*}{ Endocrine therapy } & Yes & $314(91.28)$ & $251(90.94)$ & $63(92.65)$ & \\
\hline & No & $261(85.02)$ & $208(84.90)$ & $53(85.48)$ & 0.9081 \\
\hline Trastuzumab & Yes & $46(14.98)$ & $37(15.10)$ & $9(14.52)$ & \\
\hline
\end{tabular}

and 11 in the SLND-alone group (2.5\%). In IBCSG-23-01 trial, 31 patients $(3.3 \%)$ did not receive the study treatment as randomized: 17 in the ALND arm (4\%) and 14 in the SLND-alone arm (3\%).

We reported in this study 548 patients (60.7\%) with SN macro-metastases and 298 with micro-metastases (33.0\%). Among 477 patients eligible in our study to Z0011 trial, we observed 276 macro-metastases (57.86\%) and 201 micro-metastases (42.14\%) in comparison with Z11 trial results, respectively 430 and 301 patients (50.2\% and 35.2) but with undetermined SN status for 125 patients (14.6\%). It was reported a significant difference of macro and micro-metastases between the two arms of the Z11 trial $(44.8 \%$ of micro-metastases in no ALND arm versus
37.5\% in ALND arm) with also 33 patients without involved SN, respectively 29 in no ALND arm and 4 in ALND arm. This point represents a strong limitation to demonstrated equivalent results between two arms.

Among 355 patients reported in our study, 340 (95.77\%) were eligible to IBCSG-23-01 trial: 285 with micrometastases $(83.82 \%)$ and 55 with ITC (16.18\%). We can't compare this SN status with IBCSG-23-01 trial because this repartition was not done.

In our study 84 patients (8.89\%) have negative ER and PR $(<10 \%)$ and 861 have positive ER or PR with $82.32 \%$ HR+ Her2- tumors, $5.42 \%$ triple negative tumors and $12.25 \%$ tumors Her2+ and HR- or HR+. Tumors subtypes were not done for randomized trials reported in literature.

Table 6 Multivariate analysis of factors associated with NSN involvement in patients treated by radiotherapy and without neoadjuvant chemotherapy

\begin{tabular}{|c|c|c|c|c|c|}
\hline Effect & $P$ value & Contrast & $\begin{array}{l}\text { Odd Ratio } \\
\text { Estimate }\end{array}$ & $95 \% \mathrm{Cl}$ & $P$ value \\
\hline \multirow[t]{2}{*}{ Age in class } & 0.2643 & $41-75$ vs $>75$ & 1.491 & {$[0.448 ; 4.963]$} & 0.5148 \\
\hline & & $<=40$ vs $>75$ & 0.294 & {$[0.027 ; 3.174]$} & 0.3132 \\
\hline \multirow[t]{2}{*}{ Tumor Size } & 0.9532 & $10-30$ vs $<=10$ & 0.852 & {$[0.309 ; 2.352]$} & 0.7574 \\
\hline & & $>30$ vs $<=10$ & 0.869 & {$[0.218 ; 3.469]$} & 0.8420 \\
\hline LVI & 0.4760 & Pos. Vs Neg. & 0.781 & {$[0.396 ; 1.540]$} & 0.4760 \\
\hline Capsular Effraction & 0.0227 & Yes vs No & 2.311 & {$[1.124 ; 4.753]$} & 0.0227 \\
\hline Hormonal receptors & 0.7739 & Pos. Vs Neg. & 1.206 & {$[0.336 ; 4.323]$} & 0.7739 \\
\hline HER2 Status & 0.1481 & Pos. Vs Neg. & 0.406 & {$[0.119 ; 1.378]$} & 0.1481 \\
\hline \multirow[t]{2}{*}{ Histology Type } & 0.3399 & Mixed/Other vs lobular & 0.343 & {$[0.082 ; 1.430]$} & 0.1418 \\
\hline & & Ductal vs lobular & 0.644 & {$[0.244 ; 1.699]$} & 0.3739 \\
\hline \multirow[t]{2}{*}{ SBR Grade } & 0.1191 & Gr II vs Gr I & 2.672 & {$[1.048 ; 6.817]$} & 0.0396 \\
\hline & & Gr III vs Gr I & 2.179 & {$[0.738 ; 6.436]$} & 0.1586 \\
\hline $\mathrm{Nb}$ Involved SN & 0.0808 & $>2$ vs $<=2$ & 8.159 & {$[0.773 ; 86.095]$} & 0.0808 \\
\hline \multirow[t]{2}{*}{ SN Status } & 0.3649 & Macro vs ITC & 3.303 & {$[0.404 ; 27.011]$} & 0.2651 \\
\hline & & Micro vs ITC & 2.247 & {$[0.262 ; 19.277]$} & 0.4603 \\
\hline
\end{tabular}


Table 7 Multivariate analysis of factors associated with NSN involvement in patients treated by radiotherapy and without chemotherapy prior to ALND

\begin{tabular}{|c|c|c|c|c|c|}
\hline Effect & $P$ value & Contrast & $\begin{array}{l}\text { Odd Ratio } \\
\text { Estimate }\end{array}$ & $95 \% \mathrm{Cl}$ & $P$ value \\
\hline \multirow[t]{2}{*}{ Age in class } & 0.2626 & $41-75$ vs $>75$ & 1.402 & {$[0.409 ; 4.805]$} & 0.5905 \\
\hline & & $<=40$ vs $>75$ & 0.256 & {$[0.023 ; 2.887]$} & 0.2702 \\
\hline \multirow[t]{2}{*}{ Tumor Size } & 0.9233 & $10-30$ vs $<=10$ & 0.842 & {$[0.273 ; 2.598]$} & 0.7644 \\
\hline & & $>30$ vs $<=10$ & 0.982 & {$[0.226 ; 4.263]$} & 0.9807 \\
\hline LVI & 0.3089 & Pos. Vs Neg. & 0.682 & {$[0.326 ; 1.425]$} & 0.3089 \\
\hline Capsular Effraction & 0.0381 & Yes vs No & 2.357 & {$[1.048 ; 5.302]$} & 0.0381 \\
\hline Hormonal receptors & 0.4821 & Pos. Vs Neg. & 1.647 & {$[0.410 ; 6.617]$} & 0.4821 \\
\hline HER2 Status & 0.1906 & Pos. Vs Neg. & 0.376 & {$[0.087 ; 1.626]$} & 0.1906 \\
\hline \multirow[t]{2}{*}{ Histology Type } & 0.5892 & Mixed/Other vs lobular & 0.478 & {$[0.111 ; 2.047]$} & 0.3196 \\
\hline & & Ductal vs lobular & 0.665 & {$[0.243 ; 1.818]$} & 0.4267 \\
\hline \multirow[t]{2}{*}{ SBR Grade } & 0.0650 & Gr I vs Gr I & 3.332 & {$[1.214 ; 9.143]$} & 0.0195 \\
\hline & & Gr III vs Gr I & 2.714 & {$[0.831 ; 8.862]$} & 0.0982 \\
\hline $\mathrm{Nb}$ Involved SN & 0.1171 & $>2$ vs $<=2$ & 6.797 & {$[0.618 ; 74.708]$} & 0.1171 \\
\hline \multirow[t]{2}{*}{ SN Status } & 0.3734 & Macro vs ITC & 3.737 & {$[0.451 ; 30.991]$} & 0.2219 \\
\hline & & Micro vs ITC & 2.635 & {$[0.305 ; 22.767]$} & 0.3784 \\
\hline
\end{tabular}

Abbreviations: $\mathrm{Cl}$ confidence interval

In Z11 trial 127 patients had ER and PR negative tumors (127/775: 16.4\%), 91 had ER negative tumors (91/925: 9.8\%) in IBCSG-23-01 trial and 28 had ER negative tumors (28/208: 13.5\%) in AATRM trial.

In our study, involved NSN rate was $19 \%$, respectively 15.7 and $12.8 \%$ for patients eligible to Z11 and IBCSG23-01 trials, in comparison with 27.3, 7.6 and $13 \%$ for Z11, IBCSG-23-01 and AATRM trials. The difference (11.6\%) observed between Z11 trial and our results for patients eligible to Z11 could be explain by the proportion of patients in our study with ALND performed after chemotherapy (17.9\%: 75/419). Involved NSN rate is significantly lower for patients with ALND performed after chemotherapy, with a significant down staging in comparison with others patients with chemotherapy after ALND or without chemotherapy. Similar results were observed after NAC with a $41 \%$ related NAC down staging in ACOSOG-Z1071 trial [15], 17.8\% positive NSN rate for patients $\mathrm{cN0}$ with positive SN before NAC (SENTINA trial) [16] and 40.8\% related NAC down staging for patients with positive cytology axillary node before NAC in Park et al. study [17]. This is an important observation which can in part explain the very low axillary recurrence rate for patients without cALND. A high proportion of patients received chemotherapy: $70.4 \%(671 / 953)$ in our study, $57.9 \%(496 / 856)$ in Z0011, 69.4\% (646/931) in IBCSG-23-01 and 92.1\% (199/216) in AATRM.

Tangential fields of breast irradiation have also a therapeutic impact on axillary basin as we can observe with an 10-year axillary recurrence rate of 0.08 and $0.75 \%$ respectively for WBI and partial breast irradiation (HR
0,25: 0,08-0,75) [18]. In our study 95.9\% (896/934) patients received radiotherapy on the breast or chest wall, with respectively 89.3\% (540/605) in Z11, 89.7\% (209/215 after conservative treatment) in AATRM and 80.6\% (661/820 WBI after breast conserving surgery) in IBCSG-23-01. Modality of radiotherapy is a key point in these randomized studies. In Z11, detailed radiotherapy records were obtained for only 228 patients $(26.6 \%)$, of whom 185 (81.1\%) received tangent-only treatment and 43 (18.9\%) received directed regional nodal radiotherapy using 3 or more fields for patients with greater nodal involvement (55.6\% for 3 nodes involved and $81.3 \%$ for $\geq 4$ nodes involved) and more often for patients without ALND (65\%: 13/20 in the ALND arm versus 100\%: 5/5 in the SLND arm for patients with $\geq 3$ involved nodes) [19]. This point, with about $20 \%$ of patients who received directed regional axillary radiotherapy, represents a strong limitation to demonstrated equivalent results between two arms.

Moreover, ET can also have a therapeutic impact on axillary lymph nodes [20] and was often performed: $46.5 \%$ (398/856) for Z11, 87.8\% (817/931) for IBCSG-23-01, $61.6 \%(133 / 216)$ in AATRM and $89.61 \%(673 / 751)$ in our study.

Total mastectomy was done for 86 patients (9.2\%) in IBCSG-23-01 trial, 18 (7.7\%) in AATRM and 170 (17.65\%) in our study. We reported total mastectomy for $16.6 \%$ (59/ 355) patients with SN involved by ITC or micro-metastases and $18.2 \%(100 / 548)$ for SN macro-metastases with chest wall irradiation respectively for $61.8 \%$ (34/55) and 93.9\% (92/98) of patients. No data was reported about mastectomy 
with SN macro-metastases in previous randomized studies. We hope that results of SERC trial and BOOG 2013-07 trial [21] should be a sufficient rational to propose omission of cALND for patients with SN macro-metastases with mastectomy in the next future. Unfortunately, BOOG trial had to be shut down due to insufficient inclusion.

For 289 patient's non eligible to Z11, we observed higher involved NSN rate in comparison with others patients and higher rate when cALND was performed before chemotherapy. This group of patients, which represent 30\% of all patients in our study, was not included in previous randomized trials and received more adjuvant treatments.

Several non-inferiority trials with ALND randomization for involved SN by macro-metastases are ongoing [21-24] in order to confirm the possibility to avoid ALND, considering that previous trial's results have a low level of evidence [25]. In AMAROS trial, there was no significant difference between two arms with ALND or axillary radiotherapy for patients with $\mathrm{SN}$ involvement, but authors discussed these results because no equivalent results could be achieved considering a smaller number of patients in this trial [26]. In SERC trial, we proposed to analyzed SN macro-metastases but also micro-metastases or ITC with a planned stratification between these $\mathrm{SN}$ metastases sizes, both to conservative treatment and mastectomy, because results for $\mathrm{SN}$ micro-metastases were not sufficient to consider that ALND omission is demonstrated with a high level of evidence, particularly in cases of mastectomy [27].

\section{Conclusion}

The main objective of SERC trial is to demonstrate non inferiority of cALND omission to confirm or not previous randomized trials results for the same patients but also for others patients particularly for patients with capsular effraction or after mastectomy. A strong interaction between timing of cALND and chemotherapy with positive NSN rate was observed for different sizes of SN involvement.

Despite the declining trend to indicate CALND, the rate of inclusion since 2013 seems satisfactory, with however a heterogeneous participation of the centers, and could be improved by a better participation on a large number of centers. Actually, 1834 patients had been included in SERC trial.

\section{Additional file}

Additional file 1: Figure S1. Wrong treatment received: A) according to time to study initiation. B) according to cumulative number of included patients. (PNG $32 \mathrm{~kb}$ )

\section{Abbreviations}

AC: Adjuvant chemotherapy; ALND: Axillary lymph node dissection; CALND: Complementary axillary lymph node dissection; CRFs: Case report form; ET: Endocrine-therapy; HES: Hematoxylin and eosin staining; HR: Hormonal receptor; ITC: Isolated tumor cells; IVRS: Interactive voice-response system;
LVI: Lympho vascular involvement; NAC: Neoadjuvant chemotherapy; NSN: Non-sentinel node; PMRT: Post mastectomy radiotherapy; SBR: Scarff-Bloom et Richardson; SERC: Sentinel Envahi Randomisaton Curage (essai de phase III multicentrique randomise de non-infériorité de la réalisation ou non du curage axillaire en cas d'envahissement du (des) ganglion(s) sentinelle(s) dans le cancer du sein invasif - SERC-IPC 2012-001); SLNB: Sentinel lymph node biopsy; SN: Sentinel node; WBI: Whole breast irradiation

\section{Acknowledgements \\ None.}

\section{Funding}

This research did not receive any specific grant from funding agencies in the public, commercial, or not-for-profit sectors. The French PHRC sponsored the trial. There was no pharmaceutical support.

\section{Availability of data and materials}

Data were collected from participating centers through electronic CRFs, and centralized in a common database at the Institute-Paoli-Calmettes Data-Center-Unit. All data generated or analysed during this study are included in this published article (and Additional file 1), but if not sufficient available from the corresponding author on reasonable request.

\section{Authors' contributions}

All authors confirm that they all read the manuscript and agree to its publication in BMC CANCER in this form. They assure that the manuscript is not, and will not be, under simultaneous consideration by any other publication. The paper reports previously unpublished work. All authors have been included in SERC program and have participated to the redaction of the protocol. All authors have participated to the data collection. GH and $J M B$ have written this document. All authors approve final version of paper for submission.

\section{Ethics approval and consent to participate}

All relevant ethical approvals from our institutional review board ethics committee (IPC - Comité d'Orientation Stratégique) have been obtained prior to study commencement.

Written informed consent was obtained from all patients prior to study entry.

\section{Consent for publication}

Not applicable.

\section{Competing interests}

The authors declare that they have no competing interests.

\section{Publisher's Note}

Springer Nature remains neutral with regard to jurisdictional claims in published maps and institutional affiliations.

\section{Author details}

${ }^{1}$ Institut Paoli Calmettes \& CRCM \& Aix Marseille Univ, 232 Bd de Sainte Marguerite, 13009 Marseille, France. ${ }^{2}$ Department of surgery, Institut Paoli Calmettes \& CRCM \& Aix Marseille Univ, 232 Bd Ste Marguerite, Marseille, France. ${ }^{3}$ Department of radiotherapy, Institut Paoli Calmettes \& CRCM \& Aix Marseille Univ, 232 Bd Ste Marguerite, Marseille, France. ${ }^{4}$ Department of biostatistics, Institut Paoli Calmettes \& CRCM \& Aix Marseille Univ, 232 Bd Ste Marguerite, Marseille, France. ${ }^{5}$ Institut de Cancérologie de l'Ouest - Site Paul Papin, 15 rue André Boquel, 1005949055 Angers Cedex 02, CS, France. ${ }^{6}$ Polyclinique Urbain V, Chemin du Pont des Deux Eaux, 84000 Avignon, France. ${ }^{7}$ Institut Bergonie, 229 cours de l'Argonne, 33076 Bordeaux Cedex, France. ${ }^{8}$ Centre Jean Perrin, 58 rue Montalembert BP 392, 63011 Clermont Ferrand Cedex, France. ${ }^{9} \mathrm{HME}$ CHU Dupuytren, 2 avenue Martin Luther King, 87000 Limoges, France. ${ }^{10}$ Centre Léon Bérard, 28 rue Laennec, 69373 Lyon Cedex 8, France. ${ }^{11}$ Clinique du Parc Rambot 2, Avenue du Dr Aurientis, 13100 Aix en Provence, France. ${ }^{12}$ Clinique Pasteur, 294 boulevard Charles de Gaulle, 07500 Guilherand Granges, France. ${ }^{13}$ Centre Oscar Lambret, 3 rue F.

Combemal, 59000 Lille, France. ${ }^{14}$ Hôpital Tenon, 4 rue de la Chine, 75020 Paris, France. ${ }^{15}$ Institut Jean Godinot, 1 rue du Général Koenig, 51056 Reims, France. ${ }^{16}$ Centre Clinical, 2 chemin Frégenueil CS 42510 Soyaux, 16025 
Angoulème, France. ${ }^{17}$ Hôpitaux Du Léman, 3 avenue de la Dame, 74200 Thonon, France. ${ }^{18}$ Groupe Hospitalier Des Diaconesses Croix Saint Simon, Site Reuilly, 18 rue Sergent Bauchat, 75012 Paris, France. ${ }^{19}$ Institut Universitaire du Cancer Toulouse, Oncopole, 1 avenue Irène Joliot-Curie, 31059 Toulouse, France. ${ }^{20}$ Hôpital Sainte Musse (CHITS), Service de chirurgie viscérale, Rue Henri Sainte-Claire Deville, 83056 Toulon, France. ${ }^{21} \mathrm{CH}$ Saint Quentin, 1 avenue Michel de I'Hospital, B.P. 608, 02321 Saint Quentin Cedex, France. ${ }^{22} \mathrm{GHM}$ de Grenoble, La Clinique des Eaux Claires, 8 rue du Dr Calmette, 38028 Grenoble Cedex 1, France. ${ }^{23}$ Clinique Clementville, 25 rue de Clémentville, 34070 Montpellier, France. ${ }^{24} \mathrm{CHR}$ du Pays d'Aix, Avenue des Tamaris, 13616 Aix en Provence Cedex 1, France. ${ }^{25} \mathrm{CHRU}$ Nimes, Place du Pr Robert Debré, 30029 Nimes Cedex 9, France. ${ }^{26} \mathrm{GCS}$ Recherche et Innovation Sante Sarcelles, 6 avenue Charles Peguy, 95200 Sarcelles, France. ${ }^{27}$ ICM Institut Régional du Cancer Montpellier, 208 avenue des Apothicaires - Parc Euromédecine, 34298 Montpellier Cedex 5, France.

\section{Received: 17 November 2017 Accepted: 6 November 2018} Published online: 21 November 2018

\section{References}

1. Veronesi U, Paganelli G, Viale G, Luini A, Zurrida S, Galimberti V, et al. A randomized comparison of sentinel-node biopsy with routine axillary dissection in breast cancer. N Engl J Med. 2003;349:546-53.

2. Veronesi U, Viale G, Paganelli G, et al. Sentinel lymph node biopsy in breast cancer: ten-year results of a randomized controlled study. Ann Surg. 2010; 251:595-600.

3. Krag DN, Anderson SJ, Julian TB, Brown AM, Harlow SP, Costantino JP, et al. Sentinel-lymph-node resection compared with conventional axillary-lymphnode dissection in clinically node-negative patients with breast cancer: overall survival findings from the NSABP B-32 randomised phase 3 trial. Lancet Oncol. 2010;11:927-33.

4. Lyman GH, Giuliano AE, Somerfield MR, Benson AB 3rd, Bodurka DC, Burstein $\mathrm{HJ}$, et al. American Society of Clinical Oncology guideline recommendations for sentinel lymph node biopsy in early-stage breast cancer. J Clin Oncol. 2005;23:7703-20.

5. Houvenaeghel G, Classe JM, Garbay JR, Giard S, Cohen M, Faure C, Charytansky H, Rouzier R, Daraï E, Hudry D, Azuar P, Villet R, Gimbergues P, Tunon de Lara C, Martino M, Fraisse J, Dravet F, Chauvet MP, Goncalves A, Lambaudie E. Survival impact and predictive factors of axillary recurrence after sentinel biopsy. Eur J Cancer. 2016;58:73-82.

6. Giuliano AE, McCall L, Beitsch P, Whitworth PW, Blumencranz P, Leitch AM, et al. Locoregional recurrence after sentinel lymph node dissection with or without axillary dissection in patients with sentinel lymph node metastases: the American College of Surgeons oncology group Z0011 randomized trial. Ann Surg. 2010;252(3):426-32.

7. Olson JA Jr, McCall LM, Beitsch P, and the American College of Surgeons oncology group trials Z0010 and Z0011, et al. Impact of immediate versus delayed axillary node dissection on surgical outcomes in breast cancer patients with positive sentinel nodes: results from American College of Surgeons oncology group trials Z0010 and Z0011. J Clin Oncol 2008; 26: 3530-3535.

8. Lucci A, McCall LM, Beitsch PD, and theAmerican College of Surgeons Oncology Group, et al. Surgical complications associated with sentinel lymph node dissection (SLND) plus axillary lymph node dissection compared with SLND alone in the American College of Surgeons oncology group trial Z0011. J Clin Oncol 2007; 25: 3657-3663.

9. Galimberti V, Cole BF, Zurrida S, Viale G, Luini A, Veronesi P, Baratella P, Chifu C, Sargenti M, Intra M, Gentilini O, Mastropasqua MG, Mazzarol G, Massarut S, Garbay JR, Zgajnar J, Galatius H, Recalcati A, Littlejohn D, Bamert M, Colleoni M, Price KN, Regan MM, Goldhirsch A, Coates AS, Gelber RD, Veronesi U. International Breast Cancer Study Group Trial 23-01 investigators. Axillary dissection versus no axillary dissection in patients with sentinel-node micrometastases (IBCSG 23-01): a phase 3 randomised controlled trial. Lancet Oncol. 2013;14(4):297-305.

10. Giuliano AE, Hunt KK, Ballman KV, Beitsch PD, Whitworth PW, Blumencranz PW. Leitch axillary dissection vs no axillary dissection in women with invasive breast cancer and sentinel node metastasis: a randomized clinical trial. AM, Saha S, McCall LM, morrow M. JAMA. 2011;305(6):569-75.

11. Solá M, Alberro JA, Fraile M, Santesteban P, et al. Complete axillary lymph node dissection versus clinical follow-up in breast Cancer patients with sentinel node micrometastasis: final results from the multicenter clinical trial AATRM 048/13/2000. Ann Surg Oncol. 2012;20(1):120-7.

12. Lyman GH, Temin S, Edge SB, Newman LA, Turner RR, Weaver DL, Benson AB 3rd, Bosserman LD, Burstein HJ, Cody H 3rd, Hayman J, Perkins CL, Podoloff DA, Giuliano AE. American Society of Clinical Oncology clinical practice. Sentinel lymph node biopsy for patients with early-stage breast cancer: American Society of Clinical Oncology clinical practice guideline update. J Clin Oncol. 2014;32(13):1365-83.

13. Coates AS, Winer EP, Goldhirsch A, Gelber RD, Gnant M, Piccart-Gebhart M, Thürlimann B, Senn HJ, Panel Members. Tailoring therapies-improving the management of early breast cancer: St Gallen International Expert Consensus on the Primary Therapy of Early Breast Cancer 2015. Annals of Oncology. 2015;26:1533-46.

14. Houvenaeghel G, Resbeut M, Boher JM. Sentinel node invasion: is it necessary to perform axillary lymph node dissection? Randomized trial SERC. Bull Cancer. 2014;101(4):358-63.

15. Boughey JC, Suman VJ, Mittendorf EA, Ahrendt GM, Wilke LG, Taback B, Leitch AM, Kuerer HM, Bowling M, Flippo-Morton TS, Byrd DR, Ollila DW, Julian TB, SA ML, McCallm L, Symmans WF, Le-Petross HT, Haffty BG, Buchholz TA, Nelson H, Hunt KK, Alliance for Clinical Trials in Oncology. Sentinel lymph node surgery after neoadjuvant chemotherapy in patients with node-positive breast cancer: the ACOSOG Z1071 (Alliance) clinical trial. JAMA. 2013;310(14):1455-61.

16. Kuehn T, Bauerfeind I, Fehm T, Fleige B, Hausschild M, Helms G, Lebeau A, Liedtke C, von Minckwitz G, Nekljudova V, Schmatloch S, Schrenk P, Staebler A, Untch M. Sentinel-lymph-node biopsy in patients with breast cancer before and after neoadjuvant chemotherapy (SENTINA): a prospective, multicentre cohort study. Lancet Oncol. 2013;14(7):609-18.

17. Park S, Park JM, Cho JH, Park HS, Kim SI, Park BW. Sentinel lymph node biopsy after neoadjuvant chemotherapy in patients with cytologically proven node-positive breast cancer at diagnosis. Ann Surg Oncol. 2013; 20(9):2858-65

18. Gentilini O, Botteri E, Leonardi MC, Rotmensz N, Vila J, Peradze N, Thomazini MV, Jereczek BA, Galimberti V, Luini A, Veronesi P, Orecchia R. Ipsilateral axillary recurrence after breast conservative surgery: The protective effect of whole breast radiotherapy. Radiother Oncol. 2017;122(1):37-44. https://doi. org/10.1016/j.radonc.2016.12.021 Epub 2017 Jan 4.

19. Jagsi R, Chadha M, Moni J, Ballman K, Laurie F, Buchholz TA, Giuliano A, Haffty BG. Radiation field design in the ACOSOG Z0011 (Alliance) Trial. J Clin Oncol. 2014;32(32):3600-6.

20. Early Breast Cancer Trialists' Collaborative Group (EBCTCG). Aromatase inhibitors versus tamoxifen in early breast cancer: patient-level meta-analysis of the randomised trials. Lancet. 2015;386:1341-52.

21. van Roozendaal LM, de Wilt JH, van Dalen T, van der Hage JA, Strobbe $J_{\text {, }}$ Boersma L, Linn SC, Lobbes MB, Poortmans PM, Tjan-Heijnen VC, Van de Vijver KK, de Vries J, Westenberg AH, Kessels AG, Smidt ML. The value of completion axillary treatment in sentinel node positive breast cancer patients undergoing a mastectomy: a Dutch randomized controlled multicentre trial (BOOG 2013-07). BMC Cancer. 2015;15:610. https://doi.org/ 10.1186/s12885-015-1613-2.

22. Reimer T, Hartmann S, Stachs A, Gerber B. Local treatment of the axilla in early breast cancer: concepts from the national surgical adjuvant breast and bowel project B-04 to the planned intergroup sentinel mamma trial. Breast Care. 2014;9:87-95.

23. Goyal A, Dodwell D. POSNOC: a randomized trial looking at axillary treatment in women with one or two sentinel nodes with macrometastases. Clin Oncol. 2015;27:692-5.

24. Tinterri C, Canavese G, Bruzzi P, Dozin B. SINODAR ONE, an ongoing randomized clinical trial to assess the role of axillary surgery in breast cancer patients with one or two macrometastatic sentinel nodes. Breast. 2016;30:197-200.

25. Huang T-W, Kuo KN, Chen K-H, Chen C, Hou W-H, Lee W-H, Chao T-Y, Tsai J-T, Chih-Ming S, Huang M-T, Tam K-W. Recommendation for axillary lymph node dissection in women with early breast cancer and sentinel node metastasis: a systematic review and meta-analysis of randomized controlled trials using the GRADE system. Int J Surg. 2016;34(2016):73-80.

26. Donker M, van Tienhoven G, Straver ME, Meijnen P, van de Velde CJ, Mansel RE, Cataliotti L, Westenberg AH, Klinkenbijl JH, Orzalesi L, Bouma WH, van der Mijle HC, Nieuwenhuijzen GA, Veltkamp SC, Slaets L, Duez NJ, de Graaf PW, van Dalen T, Marinelli A, Rijna H, Snoj M, Bundred NJ, Merkus JW, Belkacemi Y, Petignat P, Schinagl DA, Coens C, Messina CG, Bogaerts J, 
Rutgers EJ. Radiotherapy or surgery of the axilla after a positive sentinel node in breast cancer (EORTC 10981-22023 AMAROS): a randomised, multicentre, open-label, phase 3 non-inferiority trial. Lancet Oncol. 2014; 15(12):1303-10.

27. Houvenaeghel G, Boher JM, Reyal F, Cohen M, Garbay JR, Classe JM, Rouzier R, Giard S, Faure C, Charitansky H, Tunon de Lara C, Daraï E, Hudry D, Azuar P, Gimbergues P, Villet R, Sfumato P, Lambaudie E. Impact of completion axillary lymph node dissection in patients with breast cancer and isolated tumour cells or micrometastases in sentinel nodes. Eur J Cancer. 2016;67: 106-18. https://doi.org/10.1016/j.ejca.2016.08.003.

Ready to submit your research? Choose BMC and benefit from:

- fast, convenient online submission

- thorough peer review by experienced researchers in your field

- rapid publication on acceptance

- support for research data, including large and complex data types

- gold Open Access which fosters wider collaboration and increased citations

- maximum visibility for your research: over $100 \mathrm{M}$ website views per year

At $\mathrm{BMC}$, research is always in progress.

Learn more biomedcentral.com/submissions 